TRANSACTIONS OF THE

AMERICAN MATHEMATICAL SOCIETY

Volume 358, Number 5, Pages 1953-1970

S 0002-9947(05)04140-1

Article electronically published on December 20, 2005

\title{
SYMPLECTIC FORMS INVARIANT UNDER FREE CIRCLE ACTIONS ON 4-MANIFOLDS
}

\author{
BOGUSŁAW HAJDUK AND RAFAŁ WALCZAK
}

\begin{abstract}
Let $M$ be a smooth closed 4-manifold with a free circle action generated by a vector field $X$. Then for any invariant symplectic form $\omega$ on $M$ the contracted form $\iota_{X} \omega$ is non-vanishing. Using the map $\omega \mapsto \iota_{X} \omega$ and the related map to $H^{1}\left(M / S^{1}, \mathbb{R}\right)$ we study the topology of the space $S_{i n v}(M)$ of invariant symplectic forms on $M$. For example, the first map is proved to be a homotopy equivalence. This reduces examination of homotopy properties of $S_{i n v}$ to that of the space $\mathcal{N}_{L}$ of non-vanishing closed 1-forms satisfying certain cohomology conditions. In particular we give a description of $\pi_{0} S_{i n v}(M)$ in terms of the unit ball of Thurston's norm and calculate higher homotopy groups in some cases. Our calculations show that the homotopy type of the space of non-vanishing 1-forms representing a fixed cohomology class can be non-trivial for some torus bundles over the circle. This provides a counterexample to an open problem related to the Blank-Laudenbach theorem (which says that such spaces are connected for any closed 3-manifold). Finally, we prove some theorems on lifting almost complex structures to symplectic forms in the invariant case.
\end{abstract}

\section{INTRODUCTION}

Consider a smooth compact manifold $M$. A symplectic form on $M$ is a closed non-degenerate differential $\left(C^{\infty}\right)$ 2-form $\omega$. A manifold equipped with a symplectic form is called a symplectic manifold. Alternatively we can say that the form yields a symplectic structure on the manifold. We will always assume that $M$ is oriented and the symplectic forms are compatible with the orientation. The space of all symplectic forms on $M$ with the $C^{\infty}$ topology is, if non-empty, infinite dimensional. The main motivation for writing this paper was to get to understand the homotopy properties of this space. The simplest question here is whether this space is connected (i.e. what is the set of its connected components?). We say that two symplectic forms are homotopic if they are in the same component of the space of symplectic forms and isotopic if they are in the same component of the space of symplectic forms in a given cohomology class in $H^{2}(M, \mathbb{R})$. Thus two symplectic forms $\omega_{0}, \omega_{1}$ are homotopic if they can be joined by a continuous path of symplectic forms $\omega_{t}, t \in[0,1]$ and isotopic if there is a path such that the cohomology class of $\omega_{t}$ is constant. Hence we can ask under what conditions two given symplectic forms are homotopic (isotopic).

Received by the editors December 23, 2003.

2000 Mathematics Subject Classification. Primary 53D05; Secondary 57S25.

Key words and phrases. Circle action, symplectic form, Thurston norm.

Both authors were partially supported by Grant 2 P03A 03624 of the Polish Committee of Sci. Research.

(C) 2005 American Mathematical Society Reverts to public domain 28 years from publication 1953 
The only easy case is dimension two, when a symplectic form is an area form. Two area forms are homotopic if and only if they give the same orientation. Any two cohomologous area forms are isotopic. In higher dimensions there are very few cases for which some answers are known. In dimension 4 the problem is open even for the torus $T^{4}$. A useful and often calculable invariant is provided by almost complex structures (i.e. complex structures on the tangent bundle $T M$ ). Thus an almost complex structure is a bundle automorphism $J$ of $T M$ such that $J^{2}=-I d$. For any symplectic form $\omega$ there exist almost complex structures $J$ on $M$ tamed by $\omega$ in the sense that $\omega(V, J V)>0$ for any non-zero tangent vector $V$. The set of almost complex structures tamed by $\omega$ is a contractible infinitely-dimensional space, and for any continuous path $\omega_{t}$ there exists a path $J_{t}$ such that $J_{t}$ is tamed by $\omega_{t}$. Thus Chern classes of $(T M, J)$ are equal for $J$ 's tamed by homotopic symplectic forms. Again, some simple questions are still open. For example on $T^{4}$ there are homotopy classes of almost complex structures for which it is not known whether they are tamed by symplectic forms.

When one studies the case of the torus, it is easy to find that the space of harmonic (with respect to the canonical flat Riemannian metric) symplectic forms compatible with a fixed orientation is connected. Such forms have constant coefficients, and the non-degeneracy condition is a quadratic inequality which is easy to deal with. However, there is a larger class of symplectic forms which it is not so easy to understand and which can serve as an intermediate class between harmonic and general symplectic forms. This is the space of those symplectic forms which are invariant with respect to a linear action of the circle $S^{1}$ on the torus.

The primary aim of this paper is to study the homotopy properties of the space $S_{i n v}(M)$ of invariant symplectic forms compatible with the orientation on a closed oriented 4-manifold $M$ equipped with a free circle action.

Some theorems about existence and structure of such forms and manifolds were proved in Ba1, Bo, [FMG].

The basic observation is that a symplectic form $\omega$ on $M$ invariant under a free circle action gives a non-vanishing closed 1-form $\iota_{X} \omega$ under contraction with the infinitesimal generator $X$ of the action. Thus there is also a closed non-vanishing form having rational periods, and this implies that $M$ fibers over the circle Ti]. Moreover, since $\iota_{X} \omega$ is invariant and vanishes on the vertical bundle of the fibration by circles $\pi: M \rightarrow M / S^{1}$, there is a closed non-vanishing 1 -form $\alpha$ on $M / S^{1}$ such that $\iota_{X} \omega=\pi^{*} \alpha$. Thus the orbit space $M / S^{1}$ also fibers over $S^{1}$. This is a severe topological restriction if $M$ is closed. In dimension four it implies that $M$ is either aspherical or diffeomorphic to $S^{2} \times T^{2}$.

There is also a restriction for the cohomology class of $\alpha$ : we always have $c_{1}(\pi) \cup$ $[\alpha]=0$, where $c_{1}(\pi)$ is the first Chern class of the circle fibration $\pi$ (see Lemma 2.1). The last condition was proved in Bo, by a kind of inflation trick, to be sufficient for the existence of an invariant symplectic form determined by a given non-vanishing and closed form $\alpha$ (see (2.2) ).

In many instances the questions about the topology of the space of symplectic forms lead to the problem that natural maps have unusual properties. For example, we have the map to $H^{2}(M, \mathbb{R})$ which associates to each symplectic form its cohomology class or the map to the moduli space of symplectic forms. If this map has nice properties (for example the lifting path property), then one can apply the standard machinery of algebraic topology to study the topology of the space of 
symplectic forms. Unfortunately, examples as in MD1 show that this is not true in general, for dimensions greater than four.

In the invariant case the map $\omega \mapsto \alpha$ provides dimensional and degree reduction so the problems on invariant symplectic forms can be expressed in terms of 1-forms (cf. MT]). We show that in dimension four this map as well as the induced map to $H^{1}(N, \mathbb{R})$ have quite good homotopy properties. Our main result is that this map is a homotopy equivalence between the space of invariant symplectic forms $S_{i n v}(M)$ compatible with the given orientation and the space $\mathcal{N}_{L}$ of these non-vanishing closed 1-forms which belong to the hyperplane

$$
L=\left\{x \in H^{1}\left(N^{3}, \mathbb{R}\right): x \cup c_{1}(\pi)=0\right\}
$$

(Theorem 2.10). There are three principal ingredients in the proof. First is BlankLaudenbach's theorem which says that any two closed cohomologous non-vanishing 1 -forms on a closed oriented 3-manifold are isotopic. Second, the Thurston norm is used to decide which cohomology classes in $H^{1}(M, \mathbb{R})$ are represented by nonvanishing closed 1-forms. Finally, to pass from 1-forms to symplectic forms we use inflation formula (2.2). The main technical step is that any path of 1 -forms satisfying a necessary condition (2.1) lifts to a path of symplectic forms (Lemma 2.5). To prove this theorem we also need some kind of parametric version of Lemma 2.5. Namely, in order to use the Whitehead theorem we prove that Lemma2.5holds when 1-forms $\left\{\alpha_{x}\right\}_{x \in X}$ are parameterized by $X=S^{n-1}$ or $X=D^{n}$.

As an immediate corollary we get that the map $\omega \mapsto \alpha$ provides a bijective correspondence between $\pi_{0} S_{i n v}$ and $\pi_{0} \mathcal{N}_{L}$, which in turn can be identified with the set of these fibred codimension one faces of Thurston's norm ball whose intersection with the hyperplane $L$ is non-empty (Corollary 2.11). Another application is given in Corollary 2.12, any two symplectic forms on $T^{4}$ invariant with respect to any free circle action and determining the same orientation are homotopic through invariant symplectic forms. Any such form is also isotopic to a form with constant coefficients.

Next we use this result to give sample calculations. For the torus $T^{4}$ we prove that $S_{i n v}$ is simply connected (Corollary 3.1) and for every nontrivial circle bundle over $T^{3}$ we have $\pi_{1}\left(S_{\text {inv }}\right)=\mathbb{Z}$ (Corollary 3.2). We also show a class of closed oriented 3-manifolds for which Blank-Laudenbach's theorem does not extend to higher homotopy groups: 2 -torus fibre bundles over the circle with the monodromy

$$
A=\left(\begin{array}{cc}
1 & k \\
0 & 1
\end{array}\right), \quad k \in \mathbb{Z}
$$

For any such manifold Proposition 3.3 shows that the space of non-vanishing 1forms in a fixed cohomology class has the homotopy type of Eilenberg-MacLane space $K(\mathbb{Z}, 2)$ (assuming the class is represented by a fibration over $S^{1}$ ). Let us mention another interesting space which appears in our considerations. It is the space of automorphisms of a codimension 1 foliation on $N^{3}$. We use calculations of this space for the foliation by (compact) fibers of a fibration over $S^{1}$, but possibly computations for $S_{i n v}$ can give some information on automorphisms of foliations with non-compact leaves. If $N$ is a circle fibration over the torus $T^{2}$, we show contractibility of the space of invariant symplectic forms for which the corresponding cohomology class $[\alpha]$ is fixed (Corollary [3.5).

In the last section, we study, from the similar viewpoint, the correspondence between symplectic forms and almost complex structures in the invariant case. It is known that for a symplectic 4-manifold there exist homotopy classes of almost 
complex structures not tamed by any symplectic form. Non-trivial examples of that kind were given in [CLO. They are obtained by twisting a given $J$ in a disc and calculating the Seiberg-Witten invariant. If the given almost complex structure is tamed by a symplectic structure, then Taubes' theorem [TA1, [TA2] shows that the twisted one cannot be tamed. A similar twist can be done transversally along an orbit of any linear circle action in an invariant way, but Seiberg-Witten invariants do not change. It is not known whether the resulting almost complex structures are tamed by (maybe not invariant) symplectic forms. Nevertheless, in the invariant case, some interesting relations between the topology of almost complex structures and that of $S_{i n v}$ exist. We prove the following lifting property (Theorem 4.1). Suppose $J_{0}, J_{1}$ are tamed by invariant symplectic structures $\omega_{1}, \omega_{2}$ and there is a continuous path $J_{t}, t \in[0,1]$ of invariant almost complex structures. In general it is not possible to lift the path to a path $\omega_{t}$ that tames $J_{t}$, but we show that there is another path of almost complex structures with the same ends which does lift. In Theorem 4.6 we also give a sufficient condition for an invariant $J$ to be homotopic to one which is tamed by an invariant symplectic form.

These results may be possibly continued in two directions. One is the natural extension to locally free actions. In that case the orbit space is an orbifold and the inflation trick does work. The problem is that Blank-Laudenbach's theorem and Thurston's norm theory are known only for smooth manifolds. Another potentially interesting extension is to the space of forms that are invariant under the flow of a non-vanishing vector field. Then the topological restrictions are relaxed ( $M$ still fibers over the circle, but it may be non-aspherical). When $M$ has no locally free circle action, this can be thought of as a substitute for invariant forms. Even if we have a free circle action on $M$, such a space may be interesting as an intermediate space between invariant and general symplectic forms.

We thank Dusa McDuff for helpful discussions at the early stage of this work. We are also indebted to Jacek Świątkowski and to the referee for numerous comments on the previous version of the manuscript.

\section{HOMOTOPY CLASSIFICATION OF SYMPLECTIC FORMS INVARIANT UNDER FREE CIRCLE ACTIONS ON 4-MANIFOLDS}

Throughout the article $M$ will denote a compact oriented smooth 4-manifold with a smooth free circle action and $N \cong M / S^{1}$. The space of invariant symplectic forms consistent with the given orientation will be denoted by $S_{i n v}$.

Let $\pi: M \rightarrow N$ denote the principal $S^{1}$-fibration given by the action. For $\omega \in S_{\text {inv }}$ consider the non-vanishing closed 1-form $\alpha$ satisfying

$$
\pi^{*} \alpha=\iota_{X} \omega,
$$

where $X$ is the infinitesimal generator of the action.

Lemma 2.1 ([B] $)$. If $\omega \in S_{i n v}$, then

$$
[\alpha] \cup c_{1}(\pi)=0 .
$$

Proof. Take any connection form $\eta \in \Omega^{1}(M, \mathbb{R})$. By Chern-Weyl's theorem there exists a closed 2-form $c_{1} \in H^{2}(N, \mathbb{R})$ such that $\pi^{*} c_{1}=d \eta$, and $c_{1} \in H^{2}(N, \mathbb{R})$ is the first Chern class of the bundle $\pi$. There is also a unique 2 -form $\beta^{\prime} \in \Omega^{2}(N, \mathbb{R})$ such that $\omega-\eta \wedge \iota_{X} \omega=\pi^{*} \beta^{\prime}$. This gives $d \eta \wedge \iota_{X} \omega=\pi^{*} d \beta^{\prime}$ as required. 
Let us recall the notion of Thurston's norm ([Th; see also [MT] $)$. If $N^{3}$ is a compact, connected and oriented manifold without boundary, then for any compact oriented $n$-component surface $S=S_{1} \sqcup \cdots \sqcup S_{n}$ embedded in $N$ define

$$
\chi_{-}(S)=\sum_{\chi\left(S_{i}\right)<0}\left|\chi\left(S_{i}\right)\right| \text {. }
$$

Thurston's norm (a seminorm, in fact) on $H_{2}\left(N^{3}, \mathbb{Z}\right)$ and, by Poincaré duality, on $H^{1}\left(N^{3}, \mathbb{Z}\right)$ is given by

$$
\|\phi\|_{T}=\inf \left\{\chi_{-}(S) \mid[S]=\phi\right\} .
$$

It can be extended to $H^{1}\left(N^{3}, \mathbb{R}\right)$. Let $B_{T}=\left\{\phi:\|\phi\|_{T} \leq 1\right\}$ denote the unit ball in Thurston's norm. It is an essential property of $B_{T}$ that this is a (possibly non-compact) polyhedron in $H^{1}\left(N^{3}, \mathbb{R}\right)$. Suppose $\phi^{\prime} \in H^{1}\left(N^{3}, \mathbb{Z}\right)$ is represented by a fibration $N^{3} \rightarrow S^{1}$. Then $\phi^{\prime}$ is contained in the open cone $\mathbb{R}_{+} \cdot F$ over a face $F$ of codimension one of $B_{T}$ or $\|\cdot\|$ vanishes everywhere and $\phi^{\prime} \neq 0, F=H^{1}\left(N^{3}, \mathbb{R}\right)-\{0\}$. In these cases we say that $F$ is a fibered face of Thurston's norm ball. We will say that a closed form $\alpha$ on $N$ defines a fibration, if $\alpha=\pi^{*} d t$, where $\pi: N \rightarrow S^{1}$ is a fibration with connected fiber and $d t$ is the standard length form on $S^{1}$. If a cohomology class belongs to the open cone over a fibered face, then arbitrarily close to it there is a class represented by a form which defines a fibration.

Another crucial property of Thurston's norm is that it can be used to decide whether two given closed non-vanishing 1-forms are homotopic through closed nonvanishing 1-forms. Namely, their cohomology classes must lie in the same fibered face $B_{T}$ or the norm must vanish everywhere [Th], BL; see Lemma 2.3 below.

Let us define the subspace $L \subset H^{1}\left(N^{3}, \mathbb{R}\right)$ by

$$
L=\left\{x \in H^{1}\left(N^{3}, \mathbb{R}\right) \mid x \cup c_{1}(\pi)=0\right\} .
$$

Notation 2.2. For a given 3-dimensional manifold $N$ let us define by $\mathcal{N}$ the set of all closed and non-vanishing 1-forms, by $\mathcal{N}_{L} \subset \mathcal{N}$ the set of all closed and nonvanishing 1-forms whose cohomology classes belong to $L$, by $\mathcal{N}_{H} \subset H^{1}(N, \mathbb{R})$ the set of all cohomology classes which are represented by non-vanishing, closed 1-forms, and finally by $\mathcal{N}^{x} \subset \mathcal{N}$ the set of all non-vanishing, closed 1-forms representing $x \in H^{1}(N, \mathbb{R})$.

The correspondence $\omega \mapsto \alpha$ defines a continuous map $S_{i n v} \rightarrow \mathcal{N}_{L}$, and a large part of this section is devoted to the study of its properties. Later we will need the following.

Lemma 2.3. Assume that a closed oriented 3-manifold $N$ fibers over the circle and we are given a path $\left\{x_{t}\right\}_{t \in[0,1]} \subset \mathcal{N}_{H}$. Then there is a path $\left\{\alpha_{t}\right\}_{t \in[0,1]} \in \mathcal{N}$ such that $\left[\alpha_{t}\right]=x_{t}$ for all $t \in[0,1]$. We can also assume that $\alpha_{0}$ and $\alpha_{1}$ are prescribed.

Proof. Note that $\mathcal{N}$ is non-empty if and only if $N$ fibers over the circle. Next we note that for each $t \in[0,1]$ there exists a path $\alpha_{t}^{\prime}$ with the desired property over a neighborhood $(t-\epsilon, t+\epsilon)$ of $t$. Choose a finite subcover and glue together these paths on their overlaps using Blank-Laudenbach's theorem [BL], which states that any two cohomologous, non-vanishing and closed 1-forms on a 3-dimensional closed oriented manifold are isotopic. Isotopy here can be understood, as in the case of symplectic forms, in two ways. The first is that two non-vanishing closed cohomologous 1-forms $\alpha_{0}, \alpha_{1}$ can be joined by a path of non-vanishing closed cohomologous forms, while the second means that we have a path of diffeomorphisms $\left\{\phi_{s}\right\}_{s \in[0,1]}$ such that $\phi_{0}=$ 
$I d$ and $\phi_{t}^{*} \alpha_{0}=\alpha_{t}$. By Moser's argument [Mo, these two notions are equivalent. The isotopy $\phi_{t}$ can be used to glue together local lifts to a path with the required property. Suppose that we have two paths $\alpha_{t}^{\prime}, \alpha_{t}^{\prime \prime}$ in the interval $\left(t_{0}-\epsilon, t_{0}+\epsilon\right)$. Then there is an isotopy $\phi_{t}$ such that $\phi_{1}^{*} \alpha_{t_{0}}^{\prime}=\alpha_{t_{0}}^{\prime \prime}$. Hence the family is defined as $\alpha_{t}^{\prime}$ for $t<t_{0}-\epsilon, \phi_{\left(t-t_{0}+\epsilon\right) / \epsilon}^{*} \alpha_{t}^{\prime}$ for $t \in\left[t_{0}-\epsilon, t_{0}\right]$ and as $\alpha_{t}^{\prime \prime}$ when $t>t_{0}$. This family is continuous and satisfies our conditions. The path can be made smooth, since we can find an isotopy $\phi_{t}$ which extends smoothly by identity for $t<0$ and by $\phi_{1}$ for $t>1$.

Corollary 2.4. The map $\mathcal{N} \rightarrow \mathcal{N}_{H}: \alpha \rightarrow[\alpha]$ has the lifting property for continuous maps $S^{1} \rightarrow \mathcal{N}_{H}$.

We will now prove some technical lemmas related to the question of whether two symplectic invariant forms $\omega_{1}, \omega_{2}$ are homotopic in $S_{i n v}$ if the corresponding 1 -forms $\alpha_{1}, \alpha_{2}$ are homotopic. Recall that (see [MT]) there exist examples of nonhomotopic non-vanishing closed 1-forms on a 3-dimensional manifold $N$ which are obtained from (non-homotopic) symplectic forms on $N \times S^{1}$.

Lemma 2.5. For any compact space $X$ and any continuous map $\alpha: X \rightarrow \mathcal{N}_{L}$, there exists a continuous map $\omega: X \rightarrow S_{i n v}$ such that $\pi^{*} \alpha(t)=\iota_{X} \omega(t)$ for every $t \in X$.

Proof. The proof uses Bouyakoub's formula $\mathrm{BO}$. In the spirit of the inflation trick of Thurston Th1 and McDuff [MD2, the formula consists of enlarging a form along the foliation determined by $\operatorname{ker} \alpha$. Thus for a given form $\alpha$,

$$
\omega=\eta \wedge \pi^{*} \alpha+\pi^{*}(K \beta+\phi)
$$

is an invariant symplectic form if $\eta \in \Omega^{1}\left(M^{4}, \mathbb{R}\right)$ is a connection form for the bundle $\pi, \beta$ is a closed 2-form on $N^{3}$ such that $\alpha \wedge \beta$ is a volume form on $N^{3}, d \phi=-c_{1} \wedge \alpha$ and $K$ is a sufficiently large real number. Obviously, $\pi^{*} \alpha=\iota_{X} \omega$. Existence of $\beta$ such as above is well known $[\mathrm{Pl}],[\mathrm{Su}]$.

Later we shall also need a parameter version, for a family of 1-forms parameterized by a disk. In fact, the parametrization space can be any paracompact space.

Lemma 2.6. Let $N^{n}$ be a closed and oriented manifold. Assume that a closed and non-vanishing 1 -form $\alpha$ on $N$ is given. Then there is a closed $(n-1)$-form $\beta$ such that $\alpha \wedge \beta$ is a volume form on $N$. Equivalently, $\beta$ is a volume form on leaves of the foliation defined by $\operatorname{ker} \alpha$. Moreover, given a paracompact space $X$ and a mapping $\phi_{1}: X \rightarrow \mathcal{N}$ into the space of closed and non-vanishing 1-forms on $N$, there exists another mapping $\phi_{2}$ from $X$ into the space of closed $(n-1)$-forms on $N$ such that $\phi_{1}(x) \wedge \phi_{2}(x)$ is a volume form on $N$ for each $x \in X$.

Proof. For each $x$ there exists a closed 2-form $\beta_{x}$ such that $\phi_{1}(y) \wedge \beta_{x}$ is a volume form for $y$ in some neighborhood $U_{x}$ of $x$. For the open covering $\left\{U_{x}\right\}_{x \in X}$ we find a locally finite subcovering $\left\{U_{\xi}\right\}$ and an associated partition of unity. Convex combinations of $\beta$ 's given by the partition of unity yields a family with the desired property.

Now consider a path $\alpha_{t}$. By Lemma 2.6 there is a continuous path of closed 2 -forms $\left\{\beta_{t}\right\}_{t \in[0,1]}$ such that $\alpha_{t} \wedge \beta_{t}$ is a volume form for each $t \in[0,1]$. 
If we choose a continuous path $\phi_{t}$ such that $d \phi_{t}=-c_{1} \wedge \alpha_{t}$ then, for $K$ large enough,

$$
\omega_{t}=\eta \wedge \pi^{*} \alpha_{t}+\pi^{*}\left(K \beta_{t}+\phi_{t}\right)
$$

is an invariant symplectic form for each $t$. This completes the proof of Lemma 2.5.

Later we will need the following easy lemma.

Lemma 2.7. If $\omega, \omega^{\prime} \in S_{i n v}$ and their corresponding 1-forms $\alpha, \alpha^{\prime}$ are equal, then $\omega, \omega^{\prime}$ are homotopic in $S_{i n v}$. The same is true in the parametric case (i.e. for any pair of continuous maps $f, f^{\prime}: X \rightarrow S_{\text {inv }}$ such that the corresponding maps to $\mathcal{N}$ are equal, $f$ is homotopic to $f^{\prime}$ ).

Proof. Under our assumptions $\omega$ and $\omega^{\prime}$ can be joined in $S_{i n v}$ by the convex combination. To see this write

$$
\omega=\eta \wedge \pi^{*} \alpha+\pi^{*} \phi, \quad \omega^{\prime}=\eta \wedge \pi^{*} \alpha+\pi^{*} \phi^{\prime}
$$

for some 2 -forms $\phi, \phi^{\prime}$ on $N$. Then

$$
\begin{gathered}
\left(\lambda \omega+(1-\lambda) \omega^{\prime}\right)^{2}=\left(\eta \wedge \pi^{*} \alpha+\pi^{*}\left(\lambda \phi+(1-\lambda) \phi^{\prime}\right)\right)^{2} \\
=\lambda \omega^{2}+(1-\lambda) \omega^{\prime 2}>0
\end{gathered}
$$

More generally for cohomologous $\omega, \omega^{\prime}$ we have the following.

Lemma 2.8. Assume that $\omega$ and $\omega^{\prime}$ are cohomologous invariant symplectic forms on a 4-dimensional manifold. Then $\omega$ and $\omega^{\prime}$ are isotopic.

Proof. Let $\alpha, \alpha^{\prime}$ correspond to $\omega, \omega^{\prime}$. It follows from Blank-Laudenbach's theorem BL that there exists an isotopy $\left\{\phi_{t}\right\}$ such that $\phi_{1}^{*} \alpha^{\prime}=\alpha$ and $\phi_{0}=I d$. The path $\phi_{t}$ lifts to a path $\widetilde{\phi_{t}}$ of equivariant diffeomorphisms on $M,\left(\widetilde{\phi_{t}}\right)_{*} X=X$ (recall that by $X$ we denoted the infinitesimal generator of the action). Then

$$
\iota_{X}\left(\widetilde{\phi_{1}}\right)^{*} \omega^{\prime}=\left(\widetilde{\phi_{1}}\right)^{*} \iota_{X} \omega^{\prime}=\left(\widetilde{\phi_{1}}\right)^{*} \pi^{*} \alpha^{\prime}=\pi^{*} \phi_{1}^{*} \alpha^{\prime}=\pi^{*} \alpha=\iota_{X} \omega
$$

and $\omega,\left(\widetilde{\phi_{1}}\right)^{*} \omega^{\prime}$ are still in the same cohomology class. To finish the proof apply Lemma 2.7 and the equivariant version of Moser's theorem [Mo].

We now can prove our main results.

Theorem 2.9. Let $M^{4}$ be a closed manifold equipped with a free circle action. Assume that we are given a continuous path $\left\{x_{t}\right\}_{t \in[0,1]} \subset H^{2}\left(M^{4}, R\right)$ such that for each $t \in[0,1]$ there exists an invariant symplectic form $\omega_{t}^{\prime}$ in the class $x_{t}$. Then there exists a continuous path $\left\{\omega_{t}\right\}_{t \in[0,1]}$ of symplectic invariant forms such that $\left[\omega_{t}\right] \in x_{t}$. Moreover, the forms $\omega_{0}, \omega_{1}$ can be prescribed.

Proof. For every $t \in[0,1]$ there exists $\epsilon>0$ such that in the closed interval $[t-$ $\epsilon, t+\epsilon]$ we have a path of symplectic and invariant forms satisfying our conditions. By the standard compactness argument there exists an increasing sequence $0=$ $t_{0}<t_{1}<\ldots<t_{n}=1$ such that in each closed interval $\left[t_{i}, t_{i+1}\right]$ the given path lifts. Using the isotopy given by Lemma 2.8 we can glue these partial lifts as in the proof of Lemma 2.3. 
In higher dimensions our arguments do not work. Let us recall an example given by McDuff.

On $M=S^{2} \times S^{2} \times T^{2}$ we have a family of symplectic forms

$$
\tau_{\lambda}=\lambda \sigma_{0} \times \sigma_{1} \times \sigma_{2}, \quad \lambda \geq 1,
$$

where $\sigma_{i}$ are area forms of total area equal to 1 . If we take the diffeomorphism $\phi: M \rightarrow M$ given by the formula

$$
\phi(z, w, s, t)=\left(z, \rho_{z, t}(w), s, t\right),
$$

where $\rho_{z, t}: S^{2} \rightarrow S^{2}$ is the rotation around the axis through $z$ through the angle $2 \pi t$, then $\tau_{\lambda}$ and $\phi^{*} \tau_{\lambda}$ are isotopic when $\lambda>1$ but are not isotopic when $\lambda=1$ MD1.

Let us define on $M$ a free circle action by the formula

$$
\theta(z, w, s, t)=(z, w, \theta s, t), \quad \theta \in S^{1} .
$$

Then $\phi$ commutes with the action and $\tau_{\lambda}$ is invariant when $\sigma_{2}$ is chosen to be invariant. Next observe that we have

$$
\iota_{X} \tau_{\lambda}=\iota_{X} \phi^{*} \tau_{\lambda}=d t
$$

if $X=\frac{\partial}{\partial s}$ denotes the infinitesimal generator of the action. As in [MD1] one can check that $\tau_{\lambda}$ and $\phi^{*} \tau_{\lambda}$ are isotopic through invariant symplectic forms for $\lambda>1$, and that $\tau_{1}, \phi^{*} \tau_{1}$ are not isotopic.

It follows that the isotopy part of Lemma 2.7 does not hold in dimensions greater than 4 .

Theorem 2.10. The map

$$
\Phi: S_{i n v} \rightarrow \mathcal{N}_{L}
$$

defined by $\omega \mapsto \alpha$, is a homotopy equivalence.

Proof. We will show that the map $\Phi_{*}: \pi_{n} S_{i n v} \rightarrow \pi_{n} \mathcal{N}_{L}$ is an isomorphism for every $n$. Since the spaces here have the homotopy type of CW-complexes, we get the conclusion by Whitehead's theorem.

To see that $\Phi_{*}$ is injective assume we are given a map $\Delta:\left(S^{n}, \star\right) \rightarrow\left(S_{i n v}, \star\right)$ and a map $\delta: D^{n+1} \rightarrow \mathcal{N}_{L}$ such that $\Phi \circ \Delta=\delta \uparrow S^{n}$. By Lemma 2.6 $\delta$ has a lift $\tilde{\delta}$ to $S_{\text {inv }}$, and Lemma 2.7 gives an isotopy between $\Delta$ and $\tilde{\delta} \mid S^{1}$. Thus $\Delta$ is null-homotopic as required. A proof that $\Phi_{*}$ is onto follows directly from Lemma 2.6 .

Corollary 2.11. Assume that $M^{4}$ is a closed oriented manifold admitting an invariant symplectic form. Then $\pi_{0} S_{i n v}$ is in bijective correspondence to the set of these fibered faces of Thurston's norm ball whose intersection with $L$ is non-empty.

Proof. It follows from Theorem 2.10 and the discussion concerning Thurston's norm.

Corollary 2.12. Let $\omega$ be a symplectic form on $T^{4}$ invariant under a free linear action of $S^{1}$. Then $\omega$ is homotopic to the standard structure $\omega_{s t}=d x_{1} \wedge d y_{1}+d x_{2} \wedge$ $d y_{2}$. Furthermore, $\omega$ is isotopic to a form with constant coefficients.

Proof. If $S^{1}$ acts linearly on $T^{4}$, then the quotient space is diffeomorphic to $T^{3}$. Any non-zero class of $H^{1}\left(T^{3}\right)$ is represented by a 1 -form with constant coefficients. Since the standard generators of $H_{2}\left(T^{3}, \mathbb{Z}\right)$ are represented by tori, Thurston's 
norm equals 0 everywhere (cf. [Th $)$. We get $B_{T}=H_{2}\left(T^{3}, \mathbb{R}\right)$, and it implies that $\mathcal{N}_{L}=\mathcal{N}$ is connected. By Corollary 2.11 and Lemma 2.8, our claim follows.

Remark 2.13. By linear action we mean an action equivalent to an action of a subgroup of the torus considered as the Lie group. It follows from Poincaré's conjecture that any free action of the circle is linear. So if Perelman's proof of the conjecture is correct, then Corollary 2.12 is valid for any free circle action.

\section{SympleCtiC FORMS AND DIFFEOMORPHISMS}

The homotopy properties of $S_{i n v}$ can be calculated, in some cases, using the action of $\operatorname{Diff}_{0}$ (= diffeomorphisms isotopic to the identity) on forms. This leads us to consider maps which are defined by the action, which we refer to as evaluation maps.

In the sequel we shall use the fact that, under some assumptions, the evaluation maps are fibrations and thus exact sequences of homotopy groups are available. We will consider evaluation maps of two kinds. The first one is the map $e v: G \rightarrow X$ given, for a $G$-space $X$ and a base point $x_{0} \in X$, by the formula $e v(g)=g x_{0}$. In this case, if the action is transitive and there are local sections of $e v$, then it is a locally trivial fibre bundle over $X$. Thus it is a fibration when the base $X$ is paracompact Wh. In our cases $X$ is either a connected component of $\operatorname{Diff}(M)$, the group of $C^{2}$-diffeomorphisms of $M$, or a closed subspace of $C^{1}-$ forms on $M$ (with $C^{1}$ topology). It is always an (infinite-dimensional) manifold modelled on a separable Banach space; cf. Pa. Another type of evaluation is given by $\mathcal{M} \rightarrow Y: f \mapsto f\left(x_{0}\right)$, where $\mathcal{M}$ is a component of the space of all continuous maps from $X$ to $Y, x_{0} \in X$. It is a fibration if $X, Y$ both are compact CW-complexes; cf. [Sp].

We will calculate some homotopy groups for the torus $T^{4}$ and some $S^{1}$-bundles over $T^{3}$. These cases are relatively easy because the inclusion $T^{k} \hookrightarrow \operatorname{Diff}_{0} T^{k}$, given by translations, is a homotopy equivalence for $k \leq 3$ (see [EE], [Ha]).

Consider the map

$$
e v_{\alpha}: \operatorname{Diff}_{0} N \longrightarrow \mathcal{N}^{[\alpha]},
$$

defined by

$$
e v_{\alpha}(\phi)=\phi^{*} \alpha
$$

(by Diff 0 we denote the identity component of Diff). It is onto due to $\mathrm{BL}$ and is a locally trivial fibration with fiber $F$ equal to the space of these diffeomorphisms of $N$ which are isotopic to the identity and preserve the form $\alpha$. By the Whitehead theorem, $\mathcal{N}^{[\alpha]}$ is contractible if and only if the inclusion $\kappa: F \rightarrow \operatorname{Diff}_{0} N$ is a homotopy equivalence.

If $N$ is the 3 -dimensional torus we claim that $\mathcal{N}^{[\alpha]}$ is contractible for any closed and non-vanishing 1-form $\alpha$ defining a fibration (i.e., $\alpha=\tau^{*} d t$ for a fibration $\tau: N \rightarrow S^{1}$ and the standard 1-form $d t$ on $S^{1}$ ). This property depends only on the cohomology class of $\alpha$ by [BL]. We will call any cohomology class fibered if it is represented by such a fibered form.

Any fibration of $T^{3}$ over the circle is trivial, so we can assume $\alpha=d x_{1}$. Then $F$ consists of fiberwise diffeomorphisms which cover rotations of $S^{1}$. Any $f \in F$ is uniquely determined by two parameters: an element of the space $\operatorname{Diff}_{0} T^{2} \times$ $\Omega_{0} \operatorname{Diff}_{0} T^{2}$ and the rotation angle $\theta \in S^{1}$, where $\Omega_{0}$ denotes the space of nullhomotopic loops. It follows from Diff $T^{2} \sim T^{2}$ and the asphericity of $T^{2}$ that we have 
the following sequence of homotopy equivalences:

$$
\operatorname{Diff}_{0} T^{2} \times \Omega_{0} \operatorname{Diff}_{0} T^{2} \times S^{1} \sim T^{2} \times \Omega_{0} T^{2} \times S^{1} \sim T^{2} \times\{\star\} \times S^{1} \sim T^{3} .
$$

Furthermore, in the diagram

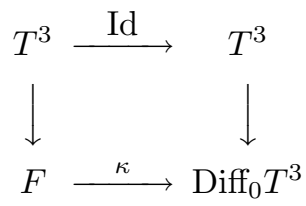

vertical maps induced by inclusions are homotopy equivalences, hence $\kappa$ is also a homotopy equivalence. Thus $\mathcal{N}^{[\alpha]}$ is contractible for a fibered class.

We now have the following corollary of Theorem 2.10,

Corollary 3.1. For any linear free action of $S^{1}$ on $T^{4}, S_{i n v}$ is simply connected.

Proof. Since in our case $\mathcal{N}_{L}=\mathcal{N}$, it is enough to prove that $\pi_{1} \mathcal{N}=0$. We will show that a given loop $\alpha: S^{1} \rightarrow \mathcal{N}$ can be deformed continuously to the subspace of non-vanishing forms with constant coefficients, which has the homotopy type of $S^{3}$. For every $t_{0} \in S^{1}, \alpha\left(t_{0}\right)$ is isotopic to a form with constant coefficients. Moreover, there exists a neighborhood $U$ of $t_{0}$ and an extension of the isotopy to a mapping

$$
\alpha:[0,1] \times U \longrightarrow \mathcal{N}
$$

such that $\alpha(0, t)=\alpha(t), \alpha(s, t)$ is an isotopy for fixed $t \in U$ and $\alpha(1, t)$ is a path of constant coefficients forms. This is given by $\alpha\left(s, t_{0}\right)+\delta(t)-\delta\left(t_{0}\right)$, where $\delta(t)$ is the form with constant coefficients in the cohomology class of $\alpha(t)$. If $U$ is small enough, then the convex combination yields an isotopy between $\alpha(t)$ and $\alpha(0, t)=\alpha\left(t_{0}\right)+\delta(t)-\delta\left(t_{0}\right)$.

If we consider a finite cover by neighborhoods as above, we come to a finite partition of $S^{1}$ by $t_{1}, \ldots, t_{k} \in S^{1}$ such that on each interval $\left[t_{i}, t_{i+1}\right]$ we have the required homotopy. Over each point $t_{i}$ there are two isotopies connecting $\alpha\left(t_{i}\right)$ to the form of constant coefficients, thus we see a loop in $\mathcal{N}^{\left[\alpha\left(t_{i}\right)\right]}$. If we perturb the loop by a form $u$ with constant coefficients, then we get a loop in $\mathcal{N}^{[\alpha+u]}$. If we choose $u$ small enough and such that $(\alpha+u)$ is a fibred class, then $\mathcal{N}^{[\alpha+u]}$ is contractible. For $u$ small enough the perturbation does not change the homotopy class of the loop, and this completes the proof.

Similar arguments apply when $M$ is a non-trivial bundle over $T^{3}$. Recall (cf. Section (2) that $\mathcal{N}_{H} \cap L=\mathbb{R}^{2} \backslash\{0\} \sim S^{1}$, since $L$ is a codimension one subspace of $H^{1}\left(T^{3}, \mathbb{R}\right)$. The homotopy described in the proof of Corollary 3.1 can be adjusted to preserve a base point. Moreover, any closed 1-form whose cohomology class lies in $\mathcal{N}_{H} \cap L$ can be approximated by a form representing a fibered class in $\mathcal{N}_{H} \cap L$ and which is in addition fibered. This is possible since the Chern class $c_{1}$ is an integer class. Thus we get the following

Corollary 3.2. $\pi_{1} S_{\text {inv }}=\mathbb{Z}$ for any non-trivial circle bundle over $T^{3}$.

In this context one can ask the following natural question: does LaudenbachBlank's theorem $\mathrm{BL}$. hold in the parametric case (i.e. do higher homotopy groups of $\mathcal{N}^{[\alpha]}$ always vanish)? In the next proposition we give a counterexample. Namely, we will show that for some torus bundles over $S^{1}$ and any 1-form $\alpha$ defining a fibration, the space $\mathcal{N}^{[\alpha]}$ has homotopy type of the classifying space of the circle $B S^{1}$. 
Let $N^{3}$ be a torus bundle over $S^{1}$ with the monodromy given by the matrix

$$
A=\left(\begin{array}{cc}
1 & k \\
0 & 1
\end{array}\right)
$$

where $k$ is a non-zero integer. Also let $\alpha$ be a closed and non-vanishing 1-form defining a fibration $\tau: N^{3} \rightarrow S^{1}$, so that $\alpha=\tau^{*} d t$.

Proposition 3.3. If $k \neq 0$, then $\mathcal{N}^{[\alpha]}$ is homotopy equivalent to the EilenbergMac Lane space $K(\mathbb{Z}, 2)$.

Proof. It is well known that the monodromy of $\tau$ is conjugated to $A$, so we can assume that it is equal to $A$ and $N=T^{2} \times[0,1] /(A x, 0) \approx(x, 1)$, where $T^{2} \times$ $\{t\}$ correspond to fibers of $\tau$. In this description generators of $\pi_{1} N$ are given by generators of $\pi_{1} T^{2}$, and the lift of the generator of $\pi_{1} S^{1}$ given by $t \mapsto(0,0, t)$. Denote the latter by $\gamma$. Consider the fibration given by (3.1):

$$
F \stackrel{\kappa}{\longrightarrow} \operatorname{Diff}_{0} N^{3} \stackrel{e v_{\alpha}}{\longrightarrow} \mathcal{N}^{[\alpha]} \text {. }
$$

Any diffeomorphism $f \in F$ induces on $S^{1}$ an orientation-preserving diffeomorphism preserving the form $d t$, hence a rotation. We get a fibration $F \stackrel{\pi^{\prime}}{\longrightarrow} S O(2)$ with fiber $F_{0}$ consisting of these $\phi \in \operatorname{Diff}_{0} N^{3}$ which preserve fibers of $\tau$. Its monodromy is given by $\phi \mapsto A \phi$, where by $A \phi$ we understand the composition of $\phi$ with the diffeomorphism of $N$ equal to $A$ on each fiber. Thus any diffeomorphism $\phi \in F_{0}$ can be identified with an isotopy of $T^{2}$ connecting a diffeomorphism $\phi_{0}$ to $A^{-1} \phi_{0} A$. For example, constant paths equal to $A^{n}$ are in $F_{0}$. The map $F_{0} \rightarrow \operatorname{Diff} T^{2}$ given by $\phi \mapsto \phi_{0}$ yields in turn a fibration of $F_{0}$ over a sum of some connected components of $\operatorname{Diff}\left(T^{2}\right)$. Its fiber is a subset (in fact, a sum of connected components) of the space of smooth loops $\Omega \operatorname{Diff}_{0} T^{2}$. Note that one can easily decide whether a loop is null-homotopic, namely by applying it to the map $\pi_{1}$ Diff $_{0} T^{2} \rightarrow \pi_{1} T^{2}$ defined by $\phi_{t} \mapsto \phi_{t}\left(x_{0}\right)$, where $x_{0} \in T^{2}$. For a loop corresponding to $\phi \in F$ the map is given by $\phi_{*}(\gamma)$. Since any $\phi \in F$ acts trivially on the homotopy, we see that the fiber of $F_{0} \rightarrow \operatorname{Diff} T^{2}$ is equal to $\Omega_{0} \operatorname{Diff}_{0} T^{2}$. It is contractible, thus $F_{0}$ is homotopy equivalent to the base. It remains to decide what are the components where $\phi_{0}$ lives. We claim that the isotopy class of $\phi_{0}$ is equal to that of a power of $A$. This gives homotopy equivalences $F_{0} \sim \operatorname{Diff}_{0} T^{2} \times \Omega_{0} \operatorname{Diff}_{0} T^{2} \times \mathbb{Z} \sim T^{2} \times \mathbb{Z}$, where the factor $\mathbb{Z}$ is generated by $[A] \in \pi_{0} \operatorname{Diff} T^{2}$.

Let $a, b$ be (standard) generators of $H_{1}\left(T^{2}\right)$ such that $A a=a$ and $A b=b+k a$. The fibration $\tau$ gives the presentation

$$
H_{1}\left(N^{3}\right) \cong\langle a, b, \gamma \mid k a\rangle \cong \mathbb{Z}_{k} \oplus \mathbb{Z}^{2},
$$

where $\gamma$ is the lift described above. The homomorphism $i_{*}: H_{1}\left(T^{2}\right) \rightarrow H_{1}\left(N^{3}\right)$ induced by inclusion is given by the formula

$$
\langle a, b\rangle \rightarrow\langle a, b, 0\rangle \text { in } \mathbb{Z} \oplus \mathbb{Z} \rightarrow \mathbb{Z}_{k} \oplus \mathbb{Z} \oplus \mathbb{Z} \cong H_{1}\left(N^{3}\right) .
$$

Any diffeomorphism $\phi \in F_{0}$ acts trivially on $H_{1}(N)$, hence for its restriction $\phi_{0}$ to $T^{2}$ (= the fiber of $\tau: N \rightarrow S^{1}$ ) we have

$$
\left(\phi_{0}\right)_{*} u \equiv u \quad \bmod \operatorname{ker} i_{*}
$$

for any $u \in H_{1}\left(T^{2}\right)$. This is the case if and only if

$$
\left(\phi_{0}\right)_{*}=\left(\begin{array}{cc}
1 & n k \\
0 & 1
\end{array}\right)
$$


since ker $i_{*}=\left\{\left(\begin{array}{c}n k \\ 0\end{array}\right) \mid n \in \mathbb{Z}\right\}$. Thus $\phi_{0}$ is isotopic to $A^{n}$.

It implies that the monodromy group of $\pi^{\prime}$ acts transitively on $\pi_{0} F_{0}$, thus $F$ is connected. So we obtain homotopy equivalences

$$
F \sim \operatorname{Diff}_{0} T^{2} \times \Omega_{0} \operatorname{Diff}_{0} T^{2} \times \mathbb{R} \sim T^{2} .
$$

Now consider $N$ as a principal $S^{1}$-bundle over $T^{2}$, given by the circle in $T^{2}$ invariant under $A$. Equivalently, $N$ can be described as the orbit space of the action of $\mathbb{Z}^{2}$ on $\mathbb{R}^{2} \times S^{1}$ given by

$$
\begin{aligned}
& (1,0)(x, y, \theta)=(x+1, y, \theta) \\
& (0,1)(x, y, \theta)=\left(x, y+1, e^{-2 \pi i k x} \theta\right) .
\end{aligned}
$$

This presentation is asymmetric, but the bundle is not. In particular, there is a bundle isomorphism, covering the identity of the base, between the bundle given by (3.2) and the one obtained from the action

$$
\begin{aligned}
& (1,0)(x, y, \theta)=\left(x+1, y, e^{-2 \pi i k y} \theta\right) \\
& (0,1)(x, y, \theta)=(x, y+1, \theta) .
\end{aligned}
$$

Denote by $\operatorname{Aut}_{0}(N)$ the set of automorphisms of the bundle which are isotopic to the identity as diffeomorphisms of $N$. It was proved by Hatcher $\mathrm{Ha}$ that the inclusion $\operatorname{Aut}_{0}(N) \subset \operatorname{Diff}_{0}(N)$ is a homotopy equivalence.

We will calculate, using Hatcher's theorem, the homotopy type of $\operatorname{Diff}_{0} N$.

Consider the fibration

$$
\pi: \operatorname{Aut}(N) \rightarrow \operatorname{Diff}\left(T^{2}\right),
$$

where $\pi$ sends $\psi \in \operatorname{Aut}(N)$ to the induced diffeomorphism of the base. The fiber $F_{\pi}$ is equal to the space $C^{1}\left(T^{2}, S^{1}\right)$ of $C^{1}$-maps with $C^{1}$-topology. Its homotopy type can be calculated using the following lemma, which gives the homotopy type of its connected component (of contractible maps).

Lemma 3.4. The evaluation map

$$
\text { ev }: C_{0}^{1}\left(X, S^{1}\right) \rightarrow S^{1}: f \mapsto f(*)
$$

is a homotopy equivalence for any compact connected manifold $X$ and any base point $* \in X$.

Proof. The map ev is a fibration with the fiber equal to $C_{0}^{1}\left((X, *),\left(S^{1}, *\right)\right)$. Since the mapping

$$
C_{0}^{1}\left((X, *),\left(S^{1}, *\right)\right) \rightarrow C_{0}^{1}((X, *),(\mathbb{R}, *)),
$$

which sends $\phi \in C_{0}^{1}\left((X, *),\left(S^{1}, *\right)\right)$ to its lift $\tilde{\phi}: X \rightarrow \mathbb{R}$, is a homeomorphism, the fiber is contractible. The lemma follows.

Since any two components of $C^{1}\left(T^{2}, S^{1}\right)$ are homeomorphic, Lemma 3.4 implies that $C^{1}\left(T^{2}, S^{1}\right) \sim S^{1} \times H^{1}\left(T^{2}, \mathbb{Z}\right)=S^{1} \times \mathbb{Z}^{2}$. Note that the factor $S^{1}$ corresponds to constant maps, hence, with our identifications, it is given by the circle action on $N$. In particular, the circle lies in the fiber $F$ of the fibration (3.1).

Now we want to calculate the homotopy groups of $\operatorname{Aut}_{0}(N)$. To this end consider the fibration

$$
\operatorname{Aut}_{0} N \rightarrow \operatorname{Diff}_{0} T^{2}
$$


which is the restriction of the fibration (3.3) to the identity component. The nontrivial part of its long homotopy exact sequence

$$
\cdots \rightarrow 0 \rightarrow \pi_{1} S^{1} \rightarrow \pi_{1} \operatorname{Aut}_{0}(N) \rightarrow \pi_{1} \operatorname{Diff}_{0} T^{2} \rightarrow \pi_{0} F_{\pi} \rightarrow \cdots
$$

gives a complete calculation once we know that the homomorphism $\pi_{1} \operatorname{Diff}_{0} T^{2} \rightarrow$ $\pi_{0} F_{\pi}$ given by monodromy is a monomorphism.

In order to compute this homomorphism we have to lift the loops $\alpha, \beta$ representing standard generators of $\operatorname{Diff}_{0}\left(T^{2}\right)$ to paths $\tilde{\alpha}, \tilde{\beta}$ in $\operatorname{Aut}(N)$. Using (3.2) we can define the lift as

$$
\alpha_{t}(x, y, \theta)=(x+t, y, \theta) .
$$

Since it commutes with the action (3.2), it hence defines a path in $\operatorname{Aut}_{0}(N)$ starting at the identity. The element of $\tilde{\alpha}_{1} \in F_{\pi}$ is given, as a map $T^{2} \rightarrow S^{1}$, by the formula

$$
\left(\theta_{1}, \theta_{2},\right) \mapsto \theta_{1}^{k}
$$

For the other path $\beta$ we get the map

$$
\left(\theta_{1}, \theta_{2},\right) \mapsto \theta_{2}^{k},
$$

since the bundle is symmetric with respect to change of factors in the base torus. We see that the map $\pi_{1} \operatorname{Diff}_{0} T^{2} \rightarrow \pi_{0} F_{\pi}$ is equal, under the identification of both groups with $\mathbb{Z}^{2}$, to $(a, b) \mapsto(a k, b k)$.

Now consider the fibration $\operatorname{Aut}_{0} N \rightarrow \operatorname{Diff}_{0} T^{2}$. Since $\operatorname{Aut}_{0}(N)$ is connected by Hatcher's theorem, we see that its fiber is equivalent to $S^{1} \times k \mathbb{Z} \times k \mathbb{Z} \subset F_{\pi}$ and $\operatorname{Aut}_{0}(N) \sim\left([0,1] \times[0,1] \times S^{1} \times k \mathbb{Z} \times k \mathbb{Z}\right) / \approx$, where

$$
\begin{aligned}
& (0, y, \theta, p, q) \approx(1, y, \theta, p+k, q), \\
& (x, 0, \theta, p, q) \approx(x, 1, \theta, p, q+k) .
\end{aligned}
$$

Thus Aut $_{0} N$ is homotopy equivalent to $S^{1} \times \mathbb{R} \times \mathbb{R}$. Another way to see this is to consider the homotopy exact sequence which gives that $\pi_{r} \mathrm{Aut}_{0}(N)$ is trivial for $r \neq 1$ and $\pi_{1} \operatorname{Aut}_{0}(N) \cong \mathbb{Z}$.

Coming back to the fibration of Corollary 3.1 we started with, we easily see that in its homotopy exact sequence the only non-trivial part is

$$
0 \rightarrow \pi_{2} \mathcal{N}^{[\alpha]} \rightarrow \mathbb{Z}^{2} \rightarrow \mathbb{Z} \rightarrow \pi_{1} \mathcal{N}^{[\alpha]} \rightarrow 0
$$

The middle homomorphism is onto, because the inclusion of the circle $S^{1}$ (corresponding to constant maps) into $\operatorname{Diff}_{0}(N)$ factorizes through $F$. Thus $\mathcal{N}^{[\alpha]}$ is homotopy equivalent to $K(\mathbb{Z}, 2)$.

Let $\mathcal{S}^{x} \subset S_{i n v}$ be the subset of all invariant symplectic forms for which the cohomology class of the associated 1 -form is equal to $x \in H^{1}(M ; \mathbb{R})$. The argument used in the proof of Theorem 2.10 gives

Corollary 3.5. Assume that $N$ is a principal $S^{1}$-bundle over $T^{2}$. If $M$ is the total space of any circle bundle over $N$ such that $[\alpha] \in L$ and $\alpha$ is fibered, then $\mathcal{S}^{[\alpha]}$ is contractible.

Higher homotopy groups of $S_{i n v}$ might be non-trivial, as the following simple example shows. 
Example 3.6. Consider the 4-dimensional torus $T^{4}$ with the circle acting on the last coordinate. Let us define the map $S^{2} \rightarrow S_{i n v}$ by

$$
\begin{gathered}
\left(\theta_{1}, \theta_{2}, \theta_{3}\right) \mapsto \theta_{1}\left(d x_{1} \wedge d x_{4}+d x_{2} \wedge d x_{3}\right)+\theta_{2}\left(d x_{2} \wedge d x_{4}+d x_{3} \wedge d x_{1}\right) \\
+\theta_{3}\left(d x_{3} \wedge d x_{4}+d x_{1} \wedge d x_{2}\right),
\end{gathered}
$$

where $\theta_{1}^{2}+\theta_{2}^{2}+\theta_{3}^{2}=1$. The map descends to a non-trivial element of $\pi_{2} \mathcal{N}_{H}$, hence it is not null-homotopic.

The classification of invariant symplectic forms in the case where $c_{1}(\xi)$ is a torsion element in $H^{2}\left(N^{3}, \mathbb{Z}\right)$ is much easier because there is a closed connection form in the fibre bundle $M \rightarrow N$.

\section{Almost complex structures}

We now apply Thurston's norm theory to prove that in the invariant case the existence of a path between two almost complex structures yields a homotopy of symplectic forms which are tamed by these structures.

Theorem 4.1. Let $M$ be a closed 4-manifold equipped with a free circle action. Assume there exist two invariant almost complex structures $J_{0}$ and $J_{1}$ tamed by some invariant symplectic forms $\omega_{0}$ and $\omega_{1}$ respectively. If there exists a path of invariant almost complex structures $\left\{J_{t}\right\}_{t \in[0,1]}$ connecting $J_{0}$ to $J_{1}$, then $\omega_{0}$ and $\omega_{1}$ lie in the same component of $S_{i n v}$ (they are homotopic through invariant symplectic forms).

Proof. As before, $X$ will denote the vector field generating the action and $\alpha_{i}=$ $\iota_{X} \omega_{i}, i=0,1$. We have on $N=M / S^{1}$ the family of vector fields $Y_{t}=\pi_{*}\left(J_{t} X\right), t \in$ $[0,1]$, such that $\alpha_{i}\left(Y_{i}\right)>0$ and for $i=0,1$ for all $x \in N$. Our problem can be reduced to the following one. Assume we are given two non-vanishing and closed 1-forms $\alpha_{0}$ and $\alpha_{1}$ on a 3-dimensional closed manifold together with a path of non-vanishing vector fields $Y_{t}$ such that $\alpha_{i}\left(Y_{i}\right)>0$ for $i \in\{0,1\}$. The question is whether $\alpha_{0}$ and $\alpha_{1}$ are homotopic through closed and non-vanishing 1-forms. We claim that the answer is positive.

Denote by $e(\alpha) \in H_{1}(N, Z)$ the Euler class of the 2-dimensional oriented subbundle $\operatorname{ker}(\alpha)$ of $T N$. Let us recall Thurston's theorem [Th] in slightly more convenient form [MT]:

Theorem 4.2. Suppose $\alpha \in H^{1}\left(N^{3}, Z\right)$ can be represented by a fibration over the circle $N^{3} \rightarrow S^{1}$. If $F \subset H^{1}\left(N^{3}, \mathbb{R}\right)$ is the codimension one face of the norm ball $B_{T}$ containing $\alpha$, then $\phi(e(\alpha))=-1$ for all $\phi \in F$.

Corollary 4.3. Let $\alpha_{0}$ and $\alpha_{1}$ be two non-vanishing and closed 1-forms on closed 3-dimensional manifold $N^{3}$. If $e\left(\alpha_{0}\right)=e\left(\alpha_{1}\right)$, then $\alpha_{0}$ and $\alpha_{1}$ are homotopic through closed and non-vanishing 1-forms.

Proof. Assume first that Thurston's norm on $N$ does not vanish identically and that $\alpha_{0}$ and $\alpha_{1}$ are in the codimension one faces $F_{0}$ and $F_{1}$, respectively. Then, for any $\phi \in F_{0}, \psi \in F_{1}$ we have $\phi(e)=\psi(e)=-1$, where $e=e\left(\alpha_{0}\right)=e\left(\alpha_{1}\right)$. Thus the functional

$$
H^{1}(N, \mathbb{R}) \rightarrow \mathbb{R}: \eta \mapsto \eta(e)
$$

restricted to either $F_{0}$ or $F_{1}$ is constant and equal to -1 . Thus $F_{0}=F_{1}$, and $\alpha_{0}$ and $\alpha_{1}$ lie in the same face. Hence they are homotopic by [Th]. 
If Thurston's norm on $N$ vanishes identically, then each non-zero class is represented by a closed and non-vanishing 1 -form, so $\alpha_{0}$ and $\alpha_{1}$ are homotopic by Lemma 2.3

If almost complex structures $J_{0}, J_{1}$ are homotopic, then the corresponding symplectic forms $\omega_{0}, \omega_{1}$ can be joined by a path of non-vanishing (not necessarily closed) 2 -forms. We have in turn a path of non-vanishing 1 -forms between $\alpha_{0}$ and $\alpha_{1}$, thus $e\left(\alpha_{0}\right)=e\left(\alpha_{1}\right)$. To finish the proof of Theorem 4.1 it is enough to apply Corollary 4.3 and Theorem 2.10 .

One may ask whether there is $\omega_{t}$ which is a lift of $J_{t}$ (i.e. $J_{t}$ is tamed by $\omega_{t}$ for each $t$ ). We will give an example of a path $J_{t}$ of invariant almost complex structures which has no lifts, but there exists another invariant path $J_{t}^{\prime}$ with the same ends which can be lifted.

Example 4.4. On the 3-dimensional torus $T^{3}$ there are closed, non-vanishing 1forms $\alpha_{0}, \alpha_{1}$ and a path $Y_{t}, t \in[0,1]$ of non-vanishing vector fields such that the following conditions are satisfied:

1. $\alpha_{i}\left(Y_{i}\right)>0$ for $i \in\{0,1\}$,

2. there is no path $\left\{\alpha_{t}\right\}_{t \in[0,1]}$ of closed, non-vanishing 1-forms connecting $\alpha_{0}$ to $\alpha_{1}$ such that $\alpha_{t}\left(Y_{t}\right)>0$ for all $t \in[0,1]$.

Having such data we will define $\omega_{0}, \omega_{1}, J_{t}$ on $T^{4}=T^{3} \times S^{1}$ invariant under the action of the $S^{1}$ factor, having the desired properties as follows. First, let $B_{t}$ denote the subbundle of $T T^{3}$ orthogonal (with respect to, say, standard Riemannian metric) to $Y_{t}$. It is oriented and of dimension 2 , hence there is a continuous path $J_{t}^{\perp}$ of complex structures on $B_{t}$. Since the space of almost complex structures compatible with a given orientation on $\mathbb{R}^{2}$ is contractible, we can assume $J_{0}^{\perp} J_{1}^{\perp}$ to be prescribed. We extend $J_{t}^{\perp}$ to an almost complex structure on $T^{3} \times S^{1}$ by putting $J X=Y_{t}$, where $X$ is the unit vector field on $S^{1}$. The forms $\alpha_{0}, \alpha_{1}$ and $\eta=d t$ give, by Corollary 2.2. invariant symplectic forms $\omega_{0}, \omega_{1}$ compatible with $J_{0}$ and $J_{1}$, respectively. Existence of a lift of $J_{t}$ would contradict condition 2 .

Denote the standard coordinates on $T^{3}$ by $x_{1}, x_{2}, x_{3}$, the coordinate on $S^{1}$ by $t$, and define

$$
\begin{gathered}
\alpha_{0}=d x_{1}, \alpha_{1}=d x_{2}, Y_{0}=\frac{\partial}{\partial x_{1}}, Y_{1}=\frac{\partial}{\partial x_{2}}, \\
\omega_{0}=d x_{1} \wedge d t+d x_{2} \wedge d x_{3}, \omega_{1}=d x_{2} \wedge d t+d x_{3} \wedge d x_{1}, \\
J_{0}^{\perp}\left(\frac{\partial}{\partial x_{2}}\right)=\frac{\partial}{\partial x_{3}}, J_{1}^{\perp}\left(\frac{\partial}{\partial x_{3}}\right)=\frac{\partial}{\partial x_{1}} .
\end{gathered}
$$

Choose an unknotted circle $S^{1} \subset D^{3} \subset T^{3}$ and a non-zero vector field tangent to it. The vector field extends to a vector field $Y_{\frac{1}{2}}$ on the torus $T^{3}$ and to a path $Y_{t}$ of vector fields such that the ends are $Y_{0}, Y_{1}$ defined above. Due to contractibility of the circle $\int_{S^{1}} \alpha=0$ for any closed 1-form $\alpha$, conditions 1 and 2 hold.

Corollary 4.5. The set of invariant symplectic forms in a fixed homotopy class of invariant almost complex structures on a closed 4-manifold is connected.

Consider the following simple-minded existence question: if $J$ is an almost complex structure, does $M$ admit a symplectic structure in the homotopy class of $J$ ? In general the answer is negative, for instance on $T^{4}$ there are almost complex structures having a non-zero first Chern class, while it is zero for those compatible with 
a symplectic structure. In the invariant case one can give an answer in terms of the quotient manifold $N$. The obvious necessary condition is the existence of a closed 1-form $\alpha \in \mathcal{N}_{L}$ such that $\alpha\left(\pi_{*} J X\right)>0$ on $N^{3} \cong M^{4} / S^{1}$. We shall prove that this condition is also sufficient. Together with Theorem 4.1 this gives a description of $\pi_{0} S_{i n v}$ as $\pi_{0}$ of a subspace of invariant almost complex structures on $M$.

Theorem 4.6. Let $M^{4}$ be a closed manifold equipped with a free circle action with infinitesimal generator $X$ and an invariant almost complex structure $J$. Assume that there exists a closed and non-vanishing 1 -form $\alpha \in \mathcal{N}_{L}$ such that $\alpha\left(\pi_{*} J X\right)>0$. Then $J$ is homotopic (through invariant almost complex structures) to an almost complex structure tamed by some invariant symplectic form.

Proof. Consider an invariant hermitian metric $\langle\cdot, \cdot\rangle$ for the complex bundle $(T M, J)$. Let $A$ be the orthogonal complement of $X$. Observe that $A$ is invariant, $J$-invariant and together with $\operatorname{Lin}_{\mathbb{R}}\{J X\}$ defines a connection $\eta$ for the principal circle bundle $M \rightarrow M / S^{1} \cong N$. It enables us to lift $\operatorname{ker} \alpha$ to a subbundle of $T M$, denoted by $S$.

Next let $\beta$ be a closed 2 -form on $N$ such that $\beta \wedge \alpha$ is non-vanishing. As in (2.2), we define an invariant form by

$$
\omega=\eta \wedge \pi^{*} \alpha+\pi^{*}(K \beta+\phi),
$$

where $K$ is large enough to guarantee that $\omega$ is symplectic. The constant $K$ will still require some modification. We do it later.

We will check that an invariant almost complex structure $J^{\prime \prime}$ is tamed by $\omega$ if the following conditions are met:

1. $\alpha\left(\pi_{*} J^{\prime \prime} X\right)>0$

2. $J^{\prime \prime}(S)=S$,

3. $\pi^{*}(K \beta+\phi)\left(W, J^{\prime \prime} W\right)>0$ for all non-zero $W \in S$,

4. $\pi_{*} J^{\prime \prime} X \in H$.

To this end we use the decomposition $T M=\operatorname{Lin}_{\mathbb{R}}\left\{X, J^{\prime \prime} X\right\} \oplus S$. By conditions 3 and 2 we get

$$
\omega\left(X, J^{\prime \prime} X\right)=\eta \wedge \pi^{*} \alpha\left(X, J^{\prime \prime} X\right)>0
$$

and

$$
\omega\left(W, J^{\prime \prime} W\right)=\pi^{*}(K \beta+\phi)\left(W, J^{\prime \prime} W\right)>0
$$

for any non-zero $W \in S$. The following equations:

$$
\begin{gathered}
\eta \wedge \pi^{*} \alpha(X, W)=0, \\
\eta \wedge \pi^{*} \alpha\left(J^{\prime \prime} X, W\right)=0, \\
\pi^{*}(K \beta+\phi)(X, W)=0, \\
\pi^{*}(K \beta+\phi)\left(J^{\prime \prime} X, W\right)=0
\end{gathered}
$$

hold for all $W \in S$. This is a consequence of the vanishing of $\alpha$ on $\pi_{*} X$ and $S$, and condition 4 above. It is easy to calculate that $\omega$ tames $J$.

We now show that $J$ can be deformed to $J^{\prime \prime}$ satisfying our conditions. First we deform $J$ to another almost complex complex structure preserving $S$. Thus we homotop $J$ to $J^{\prime}$ such that

$$
J^{\prime}(S)=S \text { and } J^{\prime} \uparrow \operatorname{Lin}\{X, J X\}=J \uparrow \operatorname{Lin}\{X, J X\} .
$$

Since in $\operatorname{ker} \eta$ we have $A \perp\{J X\}$ and (by the assumptions) $S \perp\{J X\}$, we can homotop $J$ to $J^{\prime}$ using the map $S \mapsto A$ given by the projection parallel to $J X$. We can proceed for example as follows. The tangent bundle $T M$ decomposes as 
$\operatorname{Lin}_{\mathbb{C}}\{X\} \oplus S$ and $\operatorname{Lin}_{\mathbb{C}}\{X\} \oplus A$, so $J$ orients $S$. Furthermore, whenever we choose unique orthonormal vectors $U_{A}=A^{\perp}$ and $U_{S}=S^{\perp}$ in ker $\eta$ appointed by choices of orientations, we have that $U_{A}+U_{S} \neq 0$. Then the shortest path $\left\{U_{t}\right\}_{t \in[0,1]}$ of orthonormal vectors connecting $U_{A}$ to $U_{S}$ gives the path $U_{t}^{\perp}$ of oriented, 2dimensional subbundles in $\operatorname{ker} \eta$. We induce almost complex structures on each $U_{t}^{\perp}$ from $A$ using the projection $A \mapsto U_{t}$ parallel to $J X$.

Next we immediately see that $\beta\left(\pi_{*} W, \pi_{*} J^{\prime} W\right)$ is of constant sign for nonzero $W \in S$, so replacing $\beta$ by $-\beta$ if necessary we can assume that

$$
\beta\left(\pi_{*} W, \pi_{*} J^{\prime} W\right)>0 .
$$

We also choose $K$ in (4.1) large enough to satisfy condition 3 . We now deform $J^{\prime}$ to $J^{\prime \prime}$ satisfying condition 4 .

The form $K \beta+\phi$ has maximal rank, so the two-sided kernel

$$
H=\{u \mid \forall v(K \beta+\phi)(u, v)=0\}
$$

is a one-dimensional subbundle of $T N$. Furthermore, $H$ is oriented because it is transversal to the canonically oriented bundle ker $\alpha$ by the condition $\alpha\left(\pi_{*} J^{\prime} X\right)>0$. Thus we are able to choose a section $Z$ of the bundle $H$ such that $\alpha(Z)>0$. We deform $J^{\prime}$ to $J^{\prime \prime}$ satisfying the conditions

$$
J^{\prime \prime}(S)=S \text { and } \pi_{*} J^{\prime \prime} X=Z .
$$

Both the vector fields $Z$ and $\pi_{*} J^{\prime} X$ are positive on $\alpha$, and so is the convex path $\left\{Z_{t}\right\}_{t \in[0,1]}$ connecting these fields. By transversality of $\operatorname{ker} \alpha$ and $Z$ we get that $\left\{\widetilde{Z}_{t}\right\}_{t \in[0,1]}$ are transversal to $S$ for all $t \in[0,1]$. Hence we define

$$
J_{t}^{\prime} X=\widetilde{Z_{t}},
$$

where $J_{0}^{\prime}=J^{\prime}$ and $J_{1}^{\prime}=J^{\prime \prime}$.

To complete this section we give an example of an invariant almost complex structure on $T^{4}$ which is not tamed by any invariant symplectic form, hence does not satisfy the assumption of Theorem 4.6. Almost complex structures on $T^{4}$ correspond to maps $T^{4} \rightarrow G L(4, \mathbb{R}) / G L(2, \mathbb{C}) \sim S^{2}$. Take the standard trivialization of the tangent bundle of $T^{4}=T^{3} \times S^{1}$, the standard almost complex structure $J$ and the action of the circle given by the $S^{1}$ factor. Twist $J$ on $D^{3} \times S^{1} \subset T^{3} \times S^{1}$ to correspond to the map

$$
T^{4} \rightarrow D^{3} \times S^{1} / \partial\left(D^{3} \times S^{1}\right) \stackrel{\text { projection }}{\longrightarrow} D^{3} / \partial D^{3} \stackrel{\text { Hopf }}{\longrightarrow} S^{2} .
$$

It follows directly from Corollary 2.12 that no invariant symplectic form tames the resulting almost complex structure. More generally, any invariant almost complex structure non-homotopic to the standard one cannot be tamed by an invariant symplectic form.

\section{REFERENCES}

[Ba1] S. Baldridge, Seiberg-Witten invariants of 4-manifolds with free circle actions. Commun. Contemp. Math. 3 (2001), 341-353. MR1849644 (2002d:57024)

[BL] S. Blank and F. Laudenbach, Isotopie de formes fermées en dimension trois. Inventiones Math. 54 (1979), 103-177. MR0550181 (81d:58003)

[Bo] A. Bouyakoub, Sur les fibrés principaux de dimension 4, en tores, munis de structures symplectiques invariantes et leurs structures complexes. C. R. Acad. Sci. Paris, t. 306, Série I, (1988), 417-420. MR0934608 (89f:53054) 
[CLO] F.Connolly, Lê Hông Vân, and Ono Kaoru, Almost complex structures which are compatible with Kähler or symplectic structures. Ann. Global Anal. Geom. 15 (1997), 325-334. MR.1472324 (98i:53038)

[EE] C.J. Earl, J. Eells, The diffeomorphism group of a compact Riemann surface. Bulletin of the AMS 73 (1967), 557-559. MR0212840 (35:3705)

[FMG] M. Fernández, J. W. Morgan, and A. Gray, Compact symplectic manifolds with free circle actions, and Massey products. Michigan Math. J. 38 (1991), 271-283. MR1098863 (92e:57049)

[Ha] A. E. Hatcher, Linearization in 3-Dimensional Topology. Proceedings of the International Congress of Mathematicians, Helsinki, 1978. MR0562642 (81g:57024)

[LM] F. Lalonde and D. McDuff, The classification of ruled symplectic 4-manifolds. Math. Res. Lett. 3 (1996), 769-778. MR1426534 (98b:57040)

[LLT] T.J. Li and A. Liu, Symplectic structures on ruled surfaces and generalized adjunction formula. Math. Res. Lett. 2 (1995), 453-471. MR.1355707 (96m:57052)

[MD1] D. McDuff, Examples of symplectic structures. Inventiones Math. 89 (1987), 13-36. MR0892186 (88m:58061)

[MD2] D. McDuff, From symplectic deformation to isotopy. in: First IP Lectures Series (ed. R. Stern). International Press, 1998. MR1635697(99j:57025)

[Mo] J.K. Moser, On the volume elements on manifolds. Transactions of the Amer. Math. Soc. 120 (1965), 280-296. MR0182927 (32:409)

[MS] D. Mc Duff and D. Salamon, Introduction to symplectic topology. Second edition; Clarendon Press, Oxford, 1998. MR.1698616 (2000g:53098)

[MT] C. T. McMullen and C. H. Taubes, 4-manifolds with inequivalent symplectic forms and 3-manifolds with inequivalent fibrations. Math. Res. Lett. 6 (1999), 681-696. MR1739225 (2000m:57045)

[Pa] R. Palais, Local triviality of the restriction map for embeddings. Commentarii Math. Helv. 34 (1960), 305-312. MR0123338 (23:A666)

[Pl] J. F. Plante, Foliations with measure preserving holonomy. Ann. Math. 102 (1975), 327361. MR0391125 (52:11947)

[S] I. Smith, On moduli spaces of symplectic forms. arXiv:math.SG/0012096.

[Sp] E.H. Spanier, Algebraic topology. McGraw-Hill, 1966. MR0210112 (35:1007)

[Su] D. Sullivan, A homological characterization of foliations consisting of minimal surfaces. Comment. Math. Helvetici 54 (1979), 218-223. MR0535056 (80m:57022)

[TA1] C.H. Taubes, The Seiberg-Witten invariants and symplectic forms. Math. Res. Lett. 1 (1994), 809-822. MR 1306023 (95j:57039)

[TA2] C.H. Taubes, More constraints on symplectic forms from the Seiberg-Witten invariants. Math. Res. Lett. 2 (1995), 9-13. MR1312973 (96a:57075)

[Th] W. Thurston, A norm for the homology of 3-manifolds. Mem. Amer. Math. Soc. 59 (1986), 99-130. MR0823443 (88h:57014)

[Th1] W. Thurston, Some simple examples of symplectic manifolds. Proceedings of the Amer. Math. Soc. 55 (1976), 467-468. MR0402764 (53:6578)

[Ti] D. Tischler, On fibering certain foliated manifolds over $S^{1}$. Topology 9 (1970), 153-154. MR0256413 (41:1069)

[Wh] G.W. Whitehead, Elements of Homotopy Theory. Graduate Texts in Mathematics 61, Springer, 1978. MR 0516508 (80b:55001)

Mathematical Institute, Wroceaw University, Pl. Grunwaldzki 2/4, 50-384 Wroceaw, Poland - And - Department of Mathematics and Information Technology, University of Warmia And Mazury, Żo€nierska 14A, 10-561 Olsztyn, Poland

E-mail address: hajduk@math.uni.wroc.pl

Mathematical Institute, WrocŁaw University, Pl. Grunwaldzki 2/4, 50-384 WrocŁaW, Poland - And - Institute of Mathematics of the Polish Academy of Sciences, Śniadeckich 8, 00-956 WarsaW, Poland

E-mail address: rwalc@math.uni.wroc.pl 